\title{
DIAGNÓSTICO DA CÁRIE DENTÁRIA NA INFÂNCIA: RELAÇÃO ENTRE OS ACHADOS CLÍNICOS E A PERCEPÇÃO DOS PAIS
}

\author{
DIAGNOSIS OF DENTAL CARIES IN CHILDREN: RELATION \\ BETWEEN CLINICAL FINDINGS AND PARENTS' PERCEPTION
}

\begin{abstract}
Lindsey Rayanne Vieira Grangeiro*, Beatriz Alves Gurguel França*, Clarissa Lopes Drumond**, Neusa Barros Dantas Neta**, Thiago Fonseca-Silva*, Raquel Gonçalves Vieira-Andrade*
\end{abstract}

Autor para correspondência: Thiago Fonseca-Silva - thiagofonsecasilva@gmail.com

*Faculdade de Odontologia, Centro Universitário Doutor Leão Sampaio, Juazeiro do Norte, Ceará, Brasil

**Departamento de Odontopediatria e Ortodontia, Universidade Federal de Minas Gerais, Belo Horizonte, Minas Gerais, Brasil

\section{R E S U M O}

INTRODUÇÃO: A cárie dentária é uma doença de caráter multifatorial que representa umas das doenças crônicas mais comuns do mundo. OBJETIVO: avaliar o nível de conhecimento dos pais/ responsáveis acerca da presença de cárie dentária em seus filhos, bem como associar a percepção dos mesmos com os dados objetivos de diagnóstico de cárie obtidos através de exame clínico.

MÉTODOS: Foi realizado um estudo piloto de caráter transversal com uma amostra de 38 crianças com idade entre 3 e 12 anos, atendidas pela clínica de Odontopediatria de uma instituição de ensino superior da região sul do estado do Ceará. Através do uso de questionário foram coletadas informações sobre percepção dos pais/responsáveis quanto à presença de cárie na criança, bem como aspectos sociodemográficos da família. O diagnóstico de cárie foi acessado através de exame clínico realizado por dois examinadores previamente treinados e calibrados através dos índices ICDAS-II e PUFA. Foram realizadas análises de distribuição de frequência, testes Qui-quadrado, Exato de Fisher, KolmogorovSmirnov e correlação de Pearson. RESULTADOS: A prevalência de cárie dentária foi de 78,9\%. Houve associação estatisticamente significativa entre presença de cárie avaliada através do índice ICDAS-II e a baixa percepção acerca da presença de cárie relatada pelos pais/responsáveis $(p=0,025)$. No entanto, verificou-se correlação forte entre "número de dentes cavitados na criança" e "número de dentes com cárie relatada pelos pais" $(r=0,605)$. CONCLUSÃO: A prevalência de cárie dentária foi alta e os pais/responsáveis tendem a não reconhecer os estágios iniciais da doença. No entanto, esse conhecimento aumenta à medida que aumentam o número de cavitações dentárias na criança.

PALAVRAS CHAVE: Cárie dentária. Criança. Epidemiologia. 
BACKGROUND: dental caries is a multifactorial disease that is one of the most common chronic diseases in the world. OBJECTIVE: the aim of this study was to assess the level of knowledge of parents/guardians about the presence of dental caries in their children and additionally evaluate the association between the perception with caries diagnosis obtained by clinical examination. METHODS: a cross-sectional study, pilot type, was conducted with a sample of 38 children aged between 3 and 12 years attended in the School of Dentistry, Department of Pediatric Dentistry, in the southern state of Ceara, Brazil. Through the questionnaire were collected informations about the perceptions of parents/guardians for presence of caries in children as well as sociodemographic characteristics of the family. The diagnosis of caries was evaluated through clinical examination performed by two examiners previously trained and calibrated in ICDAS-II and PUFA contents. The analyses of frequency distribution were performed.In addition, Chi-square test, Fisher's exact test, Kolmogorov-Smirnov test and Pearson correlation were used for statistical analysis. RESULTS: the prevalence of dental caries was $78.9 \%$. There was a statistically significant association between the presence of caries assessed by ICDAS-II index and low perception of dental caries reported by parents / guardians $(p=0.025)$. However, there was strong correlation between "number of teeth in children cavitation" and "number of teeth with caries reported by parents" $(r=0.605)$. CONCLUSION: The prevalence of dental caries was high and parents/persons responsible tended to not recognize initial stages of the disease. However, early recognition increased when the the child had a greater number of dental caries.

KEYWORDS: Dental Caries. Child. Epidemiology. 


\section{INTRODUÇÃO}

A cárie dentária é uma doença bucal de origem multifatorial, cujo desenvolvimento está, geralmente, relacionado aos hábitos dietéticos ricos em carboidratos fermentáveis, higiene bucal insatisfatória e presença de bactérias cariogênicas ${ }^{1-3}$. A cárie representa uma das doenças crônicas mais comuns na infância. Atinge crianças em distintas faixas etárias em todo 0 mundo 4 e pode ocasionar impacto na qualidade de vida como dor, perda do sono, além de alterações anatômicas que prejudicam a mastigação, fala e respiração $0^{5-8}$. No Brasil, o último levantamento epidemiológico nacional, realizado em 2010, indicou uma prevalência de cárie dentária de $53,4 \%$ em crianças de 5 anos de idade? Esta porcentagem distancia da meta de $90 \%$ das crianças livres de cárie estipulada pela Organização Mundial de Saúde $(O M S)^{10}$.

Sabe-se que a ocorrência de cárie dentária é comum em crianças socialmente desfavorecidas ${ }^{11,12}$. As altas taxas de prevalência estão associadas com a baixa escolaridade dos pais, baixa renda mensal familiar ${ }^{3,11}$, maior número de irmãos $^{13} \mathrm{e}$ mães jovens ${ }^{14}$. Estes determinantes sociais, por sua vez, também podem influenciar a compreensão do conhecimento que os indivíduos apresentam acerca dos problemas bucais. Pesquisas anteriores mostraram que quanto maior o nível socioeconômico da família, maior o grau de conhecimento sobre saúde bucal ${ }^{15}$.

Conhecer a percepção da população em torno dos problemas bucais é indispensável para ajudar na elaboração e reestruturação de programas educativos voltados à prevenção e promoção em saúde bucal ${ }^{16}$. O conhecimento adquirido pode ser aplicado e repassado aos filhos, e, dessa forma, pode-se evitar ou reduzir o número de problemas relacionados à saúde bucal, como a cárie dentária. Sabendo que a infância é o melhor período para a incorporação de hábitos bucais adequados ${ }^{17}$, avaliar a percepção dos pais/responsáveis acerca do conhecimento sobre a presença de cárie dentária em seus filhos é de grande relevância para implementação de propostas de saúde bucal que visam a prevenção desse tipo de doença na população infantil.
O objetivo do presente estudo foi avaliar o nível de conhecimento dos pais/responsáveis acerca da presença de cárie dentária em seus filhos, bem como associar os dados subjetivos de percepção dos pais com os dados objetivos de diagnóstico de cárie obtido através de exame clínico.

\section{METODOLOGIA}

\section{Questões Éticas}

Este estudo foi aprovado pelo Comitê de Ética em Pesquisa com Seres Humanos do Centro Universitário Doutor Leão Sampaio (UNILEÃO), sob o número de protocolo 1.289.774. Todos os participantes da pesquisa assinaram o Termo de Consentimento Livre e Esclarecido e o Termo de Assentimento da criança.

\section{Desenho de estudo e amostra}

O presente estudo piloto, de carácter transversal analítico, foi realizado com uma amostra de conveniência composta por 38 crianças atendidas na Clínica de Odontologia Infantil do Curso de Odontologia do Centro Universitário Doutor Leão Sampaio - UNILEÃO, na cidade de Juazeiro do Norte, Ceará, Brasil. Foram incluídas no estudo crianças de ambos os sexos com idade entre 3 e 12 anos, que procuraram atendimento na instituição no período de agosto a dezembro de 2015. Foram excluídas as crianças cujos pais/responsáveis não souberam responder às perguntas do questionário e aquelas cujas informações acerca dos exames clínicos anotados nos prontuários estavam ausentes e/ou incompletos.

\section{Coleta de Dados}

Os pais/responsáveis que aceitaram participar da pesquisa responderam individualmente a um questionário estruturado aplicado em uma sala previamente reservada e sob supervisão dos pesquisadores que os orientavam e esclareciam possíveis dúvidas em relação ao preenchimento do mesmo. Foram coletadas informações sobre a percepção dos pais/responsáveis em relação à presença/ausência de cárie dentária, hábitos de higiene bucal, histórico de dor de dente da criança, bem como o acesso prévio a serviço de saúde odontológica. Além disso, foram obtidas 
informações sobre idade, gênero, problemas de saúde da criança e dados sociodemográficos da família (escolaridade dos pais, número de filhos, renda mensal familiar, número de pessoas que viviam da renda familiar).

Após a aplicação do questionário aos pais/ responsáveis, as crianças foram encaminhadas à clínica odontológica para realização do exame clínico dentário. $O$ diagnóstico de cárie foi aferido com o auxílio de espátula de madeira, com o dente limpo, seco e bem iluminado (luz artificial), de acordo com os índices International Caries Detection and Assessment System (ICDAS-II) ${ }^{18}$ e PUFA ${ }^{19}$. Os exames dentários foram realizados por dois pesquisadores (RGVA e TFS) previamente treinados e calibrados (kappa interexaminador $=0,89 \mathrm{e}$ kappa intraexaminador $=0,91$ ).

A partir do ICDAS-II foi possível observar a presença/ausência de cárie dentária, incluindo as lesões de mancha branca que configuram os estágios iniciais da doença. Além disso, também foram coletadas informações acerca do número de dentes cavitados (mensurado a partir do número total de dentes cavitados) e a severidade da cárie dentária, categorizada como livre de cárie (ceo-d $=0$ ), baixa severidade (ceo-d $<6$ ) e alta severidade (ceo-d $\geq 6$ ). A partir do índice PUFA foi possível coletar informações acerca de dentes com envolvimento pulpar, ulcerações provocadas por deslocamento de dentes ou fragmentos de dentes, fístula e abcesso ${ }^{19}$.

Análise dos Dados
Todos os dados obtidos foram digitados, tabulados e analisados no programa Statistical Package for Social Sciences (SPSS para Windows, versão 20,0, SPSS Inc. Chicago, IL, EUA). A análise descritiva das frequências absolutas e relativas foi realizada para todas as variáveis do estudo. Os testes Quiquadrado e Exato de Fisher foram realizados para verificar a associação entre a presenço/ausência de cárie dentária na criança, avaliada através do ICDAS-II, e cada uma das variáveis independentes. Verificou-se a normalidade dos dados contínuos utilizando o teste Kolmogorov-Smirnov, além do teste de correlação de Pearson para observar a associação entre o número de dentes cavitados, número de dentes cavitados relatados pelos pais e o número de dentes com PUFA.

\section{RESULTADOS}

Dos 38 pacientes selecionados para o estudo, 22 $(57,89 \%)$ correspondiam ao gênero feminino e $16(42,11 \%)$ ao gênero masculino, com média de idade de 7,9 anos (desvio padrão= 2,11 anos). Em relação à renda familiar mensal, a maioria dos participantes da pesquisa $(52,6 \%)$ relataram receber menos de dois salários mínimos mensais. A tabela 1 exibe os resultados das análises descritivas realizadas nas variáveis categóricas do presente estudo. A tabela 2 exibe os resultados das análises descritivas realizadas nas variáveis contínuas do presente estudo.

Tabela 1. Análise de frequência das principais variáveis investigadas no estudo.

\begin{tabular}{lrc}
\hline Variáveis & N & $\%$ \\
\hline Gênero do responsável & 29 & 76,3 \\
Feminino & 9 & 23,7 \\
Masculino & & \\
Grau de parentesco do responsável & 26 & 68,4 \\
Mãe & 6 & 15,8 \\
Pai & 3 & 7,9 \\
Irmão & 3 & 7,9 \\
Tia & & \\
& & 31,6 \\
Estado civil do responsável & 12 & 63,2 \\
Solteiro (a) & 24 & 5,3 \\
Casado (a) & 2 & \\
União estável & &
\end{tabular}




\begin{tabular}{|c|c|c|}
\hline Variáveis & $\mathbf{N}$ & $\%$ \\
\hline \multicolumn{3}{|l|}{ Número de filhos } \\
\hline 0 & 3 & 7,9 \\
\hline 1 & 8 & 21,1 \\
\hline 2 & 12 & 31,6 \\
\hline$\geq 3$ & 11 & 39,4 \\
\hline \multicolumn{3}{|l|}{ Renda mensal familiar } \\
\hline$<1 S M$ & 9 & 23,7 \\
\hline 1 a $2 S M$ & 20 & 52,6 \\
\hline 2 a $4 S M$ & 8 & 21,1 \\
\hline$>5 \mathrm{SM}$ & 1 & 2,6 \\
\hline \multicolumn{3}{|c|}{ Pessoas que vivem da renda } \\
\hline Até 3 pessoas & 11 & 28,9 \\
\hline 4 pessoas & 15 & 39,5 \\
\hline$\geq 5$ pessoas & 12 & 31,6 \\
\hline \multicolumn{3}{|c|}{ Motivo da visita da criança ao dentista } \\
\hline Relacionados à cárie & 31 & 81,6 \\
\hline Queda ou trauma & 1 & 2,6 \\
\hline Prevenção & 6 & 15,8 \\
\hline \multicolumn{3}{|c|}{ Número de escovações diárias } \\
\hline Nenhuma & 1 & 2,6 \\
\hline $1 x$ & 5 & 13,2 \\
\hline $2 x$ & 20 & 52,6 \\
\hline$\geq 3 x$ & 12 & 31,6 \\
\hline \multicolumn{3}{|c|}{ Acompanhamento de escovação } \\
\hline Sim & 22 & 57,9 \\
\hline Não & 16 & 42,1 \\
\hline
\end{tabular}

Tabela 2. Análise de frequência das principais variáveis contínuas investigadas no estudo.

\begin{tabular}{lcc}
\hline Variáveis & Média & Desvio padrão \\
\hline Idade do responsável & 34,9 & 7,5 \\
Idade da criança & 7,9 & 2,11 \\
Número de dentes cavitados & 4,1 & 4,1 \\
Dentes restaurados & 0,7 & 1,4 \\
Dentes com PUFA & 0,5 & 1,0 \\
Placa visível & 55,6 & 30,1 \\
Sangramento gengival & 7,9 & 8,6
\end{tabular}

A prevalência de cárie dentária obtida a partir do índice ICDAS-II foi de 78,9\%. Através da análise bivariada verificou-se associação estatisticamente significativa entre a presença de cárie avaliada através do ICDAS-II e a presença de cárie relatada pelo pai $(p=0,025)$, sendo que $87,1 \%(n=27)$ dos pais afirmaram que a criança não tinha cárie quando na realidade as mesmas apresentavam cárie de acordo com o ICDAS-II. As demais variáveis não se associaram significativamente com $\circ$ a presença de cárie obtida através do diagnóstico do ICDAS-II (Tabela 3). 
Tabela 3. Associação entre as variáveis independentes e a presença de cárie dentária diagnosticada através do ICDAS-II.

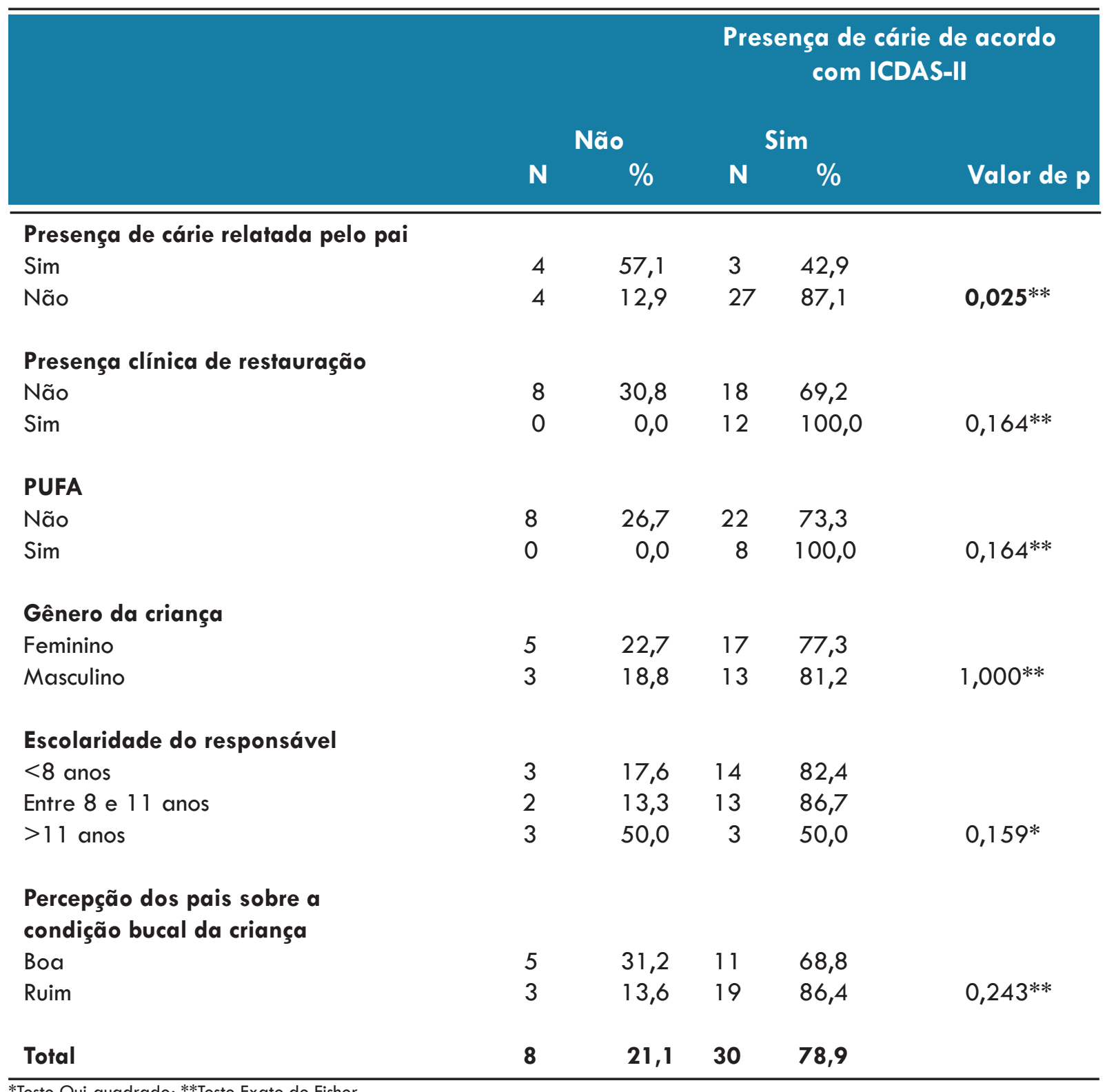

*Teste Qui-quadrado; **Teste Exato de Fisher

Através da análise de correlação de Pearson, verificou-se correlação moderada entre "número de dentes cavitados" com o "número de dentes com PUFA" $(r=0,429)$, bem como entre "número de dentes com PUFA" e "número de dentes com cárie relatada pelos pais" ( $r=0,344)$. Por outro lado, foi observada a correlação forte entre "número de dentes cavitados" e "número de dentes com cárie relatada pelo pai" $(r=0,605)$ (Tabela 4$)$.

Tabela 4. Tabela de correlação entre o número de dentes cavitados, número de dentes com PUFA e número de dentes cavitados relatado pelo pai/responsável.

\begin{tabular}{lcc}
\hline & $\begin{array}{c}\text { Número } \\
\text { Dentes } \\
\text { Cavitados }\end{array}$ & $\begin{array}{c}\text { Número } \\
\text { de dentes } \\
\text { com PUFA }\end{array}$ \\
\hline $\begin{array}{l}\text { Número Dentes Cavitados } \\
\text { Número de dentes com PUFA } \\
\text { Número de dentes com cárie } \\
\text { relatada pelo pai }\end{array}$ & 1 &, $429 *$ \\
\hline
\end{tabular}

Correlação de Pearson

*Correlação forte; *Correlação moderada 


\section{DISCUSSÃO}

No presente estudo a prevalência de cárie dentária foi alta entre as crianças. Este valor de prevalência é superior ao observado em outros estudos realizados com crianças brasileiras de faixa etária semelhante ${ }^{16,20}$. A possível explicação para - alto valor encontrado neste estudo pode estar relacionada ao fato da amostra ser proveniente de um centro de referência no atendimento odontológico de crianças. Dessa forma, já é esperada que crianças que busquem esse tipo de serviço apresentem uma grande prevalência de problemas bucais, dentre eles a cárie dentária. Além disso, o valor de prevalência pode ter sofrido interferência pela utilização do ICDAS-II. Este é um instrumento que avalia os estágios iniciais da cárie sobre o esmalte (mancha branca), além de lesões em dentina, ao contrário do índice de ceo-d e CPO-D que considera, somente, lesões cavitadas ${ }^{16,18}$.

De acordo com os resultados observados, a maioria dos pais/responsáveis apresentou baixa percepção acerca da presença de cárie dentária das crianças quando comparado com o diagnóstico clínico obtido através do ICDAS-II. O presente estudo também demonstrou que $\bigcirc$ número de dentes com cárie relatada pelo pai apresentou uma forte correlação positiva com número de dentes cavitados diagnosticado através do exame clínico. Estudo anterior relata que a percepção dos pais em relação à presença de cárie está associada com a presença de cavitação nos dentes da criança ${ }^{21}$. Sugere-se que essa percepção esteja relacionada ao fato de que lesões de cárie dentária cavitadas são mais facilmente identificáveis do que as lesões não cavitadas ${ }^{2}$. Deste modo, é importante salientar que o índice ICDAS-II, utilizado no presente estudo, considera em sua avaliação as lesões de cárie desde os seus estágios iniciais, como as lesões de mancha branca ${ }^{18}$. Assim, o baixo conhecimento dos pais acerca da presença de cárie na criança quando comparado ao diagnóstico clínico do ICDAS-II pode estar relacionado ao baixo reconhecimento acerca das lesões em seus estágios iniciais. Já quando se considera apenas o número de cavitações dentárias, a percepção dos pais/responsáveis em torno do reconhecimento da cárie dentária aumenta à medida que aumenta o número de dentes cavitados na criança.
De acordo com Gross e Howard (2001)22, os pais/cuidadores de pré-escolares e escolares são responsáveis pelas tomadas de decisão acerca dos cuidados com os problemas de saúde. Dessa forma, a percepção dos responsáveis acerca das condições bucais da criança podem determinar 0 status de saúde bucal das mesmas, bem como influenciar na procura pelo tratamento odontológico ${ }^{23,25}$. De acordo com estudos prévios, a percepção dos cuidadores pode variar de acordo com a idade da criança, grupo racialetnia, renda familiar e com o nível de escolaridade ${ }^{26,27}$. Além disso, os hábitos precários de saúde bucal dos responsáveis também podem refletir na má condição bucal da criança ${ }^{28}$.

Os resultados do presente estudo sugerem a necessidade de elaboração de estratégias de saúde públicas, como programas educativos/preventivos, que melhor orientem os pais/responsáveis acerca da identificação das lesões de cárie em seus estágios iniciais. Além disso, é fundamental a incorporação de ações preventivas que evitem a progressão dessas lesões e que contribuam de forma eficaz para uma menor incidência de cárie dentária em crianças $^{29}$. Segundo a Organização Mundial da Saúde, a implantação de estratégias de saúde pública contribui para a promoção de saúde e prevenção de problemas bucais na população ${ }^{30}$.

Até ○ presente momento, poucos estudos têm explorado os atributos, atitudes, conhecimentos e crenças dos pais em relação à ocorrência de cárie dentária em seus filhos ${ }^{15}$. Assim, mais estudos devem ser desenvolvidos acerca desse assunto. A comunidade científica e os órgãos públicos de saúde precisam ter dimensão desse conhecimento, além de verificar, posteriormente, se as formas de aplicação de políticas de prevenção são eficazes. O real conhecimento dos pais/responsáveis acerca da saúde bucal da criança poderá contribuir para a diminuição da prevalência da cárie dentária na infância.

A principal limitação desta pesquisa está relacionada ao desenho de estudo com caráter transversal e à amostra de conveniência. Em estudos dessa natureza, a exposição e o desfecho são coletados em um único momento, não sendo possível determinar a relação de causa-efeito entre as variáveis investigadas. Além disso, estudos futuros com amostras representativas são necessários para garantir a validade interna 
do estudo. Há também a possibilidade de viés de memória, visto que algumas informações foram obtidas através dos questionários administrados aos pais/responsáveis das crianças. Deste modo, outros trabalhos com amostras representativas e desenhos epidemiológicos mais elaborados com o objetivo de melhor esclarecer os resultados do presente estudo são estimulados.

\section{CONCLUSÃO}

Os resultados deste estudo revelam a prevalência de cárie dentária nas crianças examinadas foi alta. Além disso, a maioria dos pais/responsáveis desconhece a existência das lesões de mancha branca de cárie dentária, características dos estágios iniciais da doença. Esse conhecimento tende a aumentar à medida que aumentam o número de cavitações dentárias na criança.

\section{REFERÊNCIAS}

1. Fejerskov $O$. Changing paradigms in concepts on dental caries: consequences for oral health care. Caries Research. 2004;38(3):182-91. doi: $10.1159 / 000077753$

2. Fejerskov O. Concepts about dental caries and consequences for understanding the disease. Community. Dentistry and Oral Epidemiology. 1997;25(1):5-12. doi: $10.1111 /$ i.16000528.1997.tb00894.x

3. Harris $R$, Nicoll $A D$, Adair PM, Pine CM. Risk factors for dental caries in young children: a systematic review of the literature. Community Dent Health. 2004;21(1 Suppl):71-85.

4. Stecksen-Blicks C, Sunnegardh K, Borssen

E. Caries experience and background factors in 4-year-old children: time trends 1967 2002. Caries Res. 2004;38(2):149-55. doi:

\section{$10.1159 / 000075939$}

5. Abanto J, Carvalho TS, Mendes FM, Wanderley MT, Bönecker M, Raggio DP. Impact of oral diseases and disorders on oral health-related quality of life of preschool children. Community Dent Oral Epidemiol. 2011 ;39:105-114. doi: $10.1111 / \mathrm{j} .1600-0528.2010 .00580 . x$

6. Martins-júnior PA, Vieira-Andrade RG, CorrêaFaria P, Oliveira-Ferreira F, Marques LS, RamosJorge ML. Impact of early childhood caries on the oral health-related quality of life of preschool children and their parents. Caries Res. 2013;47(3):21 1-8. doi: 10.1159/000345534

7. Ramos-Jorge J, Pordeus IA, Ramos-Jorge $M L$, Marques LS, Paiva SM. Impact of untreated dental caries on quality of life of preschool children: different stages and activity. Community Dent Oral Epidemiol. 2014;42(4):311-322. doi: 10.1111/ cdoe. 12086

8. Ramos-Jorge J, Alencar BM, Pordeus IA, Soares ME, Marques LS, Ramos-Jorge ML et al. Impact of dental caries on quality of life among preschool children: emphasis on the type of tooth and stages of progression. Eur J Oral Sci. 2015;1 23(2):88-95. doi: 10.1111 /eos. 12166

9. Brasil. Ministério da Saúde. Secretaria de Atenção à Saúde/Secretaria de Vigilância em Saúde. Departamento de Atenção Básica. SB Brasil 2010: Pesquisa Nacional de Saúde Bucal resultados principais. Brasília. DF. 2010.

10. OMS (Organização Mundial da Saúde). Levantamento Epidemiológico Básico em Saúde Bucal. Manual de Instruções. 3. ed. São Paulo: Editora Santos; 1991.

11. Corrêa-Faria P, Martins-Júnior PA, VieiraAndrade RG, Marques LS, Ramos-Jorge ML. Factors associated with the development of early childhood caries among Brazilian preschoolers. Braz Oral Res. 2013;27(4):356-62. doi: 10.1590/ S1806-83242013005000021

12. Oliveira LB, Sheiham A, Bönecker M. Exploring the association of dental caries with social factors and nutritional status in Brazilian preschool 
children. Eur J Oral Sci. 2008; 116 (1):37-43. doi: $10.1111 /$ i.1600-0722.2007.00507.x

13. Wellappuli N, Amarasena N. Influence of family structure on dental caries experience of preschool children in Sri Lanka. Caries Res. 2012;46(3):208-12. doi: 10.1159/000337399

14. Niji R, Arita K, Abe Y, Lucas ME, Nishino M, Mitome M. Maternal age at birth and other risk factors in early childhood caries. Pediatr Dent. 2010;32(7):493-8.

\section{Akpabio A, Klausner CP, Inglehart MR.} Mothers'/Guardians' knowledge about promoting children's oral health. Journal of Dental Hygiene. 2008;82(1):12.

16. Gomes MC, Clementino MA, Pinto-Sarmento TC, Costa EM, Martins CC, Granville-Garcia AF, et al. Parental Perceptions of Oral Health Status in Preschool Children and Associated Factors. Braz Dent J. 2015;26(4):428-34. doi: 10.1590/01036440201300245

17. Castilho AR, Mialhe FL, Barbosa Tde S, PuppinRontani RM. Influence of family environment on children's oral health: a systematic review. J Pediatr. 2013;89(2):1 16-23. doi: 10.1016/i. iped.2013.03.014

18. Shoaib L, Deery C, Ricketts DN, Nugent ZJ. Validity and reproducibility of ICDAS II in primary teeth. Caries Research 2009;43(6):442-8. doi: $10.1159 / 000258551$

19. Monse B, Heinrich-Weltzien R, Benzian $H$, Holmgren $C$, van Palenstein Helderman W. PUFA -- an index of clinical consequences of untreated dental caries. Community Dent Oral Epidemiol. 2010;38(1):77-82. doi: $10.1111 /$ j.16000528.2009.00514.x

20. Traebert J, Suarez CS. Onofri DA, Marcenes W. Prevalence and severity of dental caries and treatment needs in small Brazilian counties. Cad Saúde Pública. 2002;18(3):817-21. doi: 10.1590/ S0102-311X2002000300030

21. Sohn W, Taichman LS, Ismail Al, Reisine S. Caregiver's perception of child's oral health status among low-income African Americans. Pediatr Dent. 2008;30(6):480-7.

22. Gross GJ, Howard M. Mothers' decision-making processes regarding health care for their children. Public Health Nurs. 2001 ; 18(3):157-68. Doi: 10.1046/i.1525-1446.2001.00157.x

23. Adair $P M$, Pine $C M$, Burnside $G$, Nicoll $A D$, Gillett $A$, Anwar $S$ et al. Familial and cultural perceptions and beliefs of oral hygiene and dietary practices among ethnically and socioeconomically diverse groups. Community Dent Health. 2004;21(1 Suppl):102-11.

\section{Mattila $M L$, Rautava $P$, Sillanpää $M$,} Paunio P. Caries in five-year-old children and associations with family-related factors. Journal of Dental Research. 2000;79(3):875-81. doi: $10.1177 / 00220345000790031501$

25. Mattila $M L$, Rautava $P$, Aromaa $M$, Ojanlatva $A$, Paunio $P$, Hyssälä $L$, et al. Behavioural and demographic factors during early childhood and poor dental health at 10 years of age. Caries Research. 2005;39(2):85-91. doi: $10.1159 / 000083152$

26. Al Agili De, Bronstein JM, Greene-mclntyre $M$. Access and utilization of dental services by Alabama Medicaidenrolled children: A parent perspective. Pediatr Dent. 2005;27(5):414-21.

27. Vargas GM, Ronzio GR. Relationship between children's dental needs and dental care utilization: United States, 1988-1994. Am J Public Health. 2002;92(11):1816-21.

28. Amin MS, Harrison RL. Understanding parents' oral health behaviors for their young children. Qualitative Health Research. 2009;19(1):1 16-27. doi: $10.1177 / 1049732308327243$

29. Casagrande L, Seminario AT, Correa MB, Werle SB, Maltz M, Demarco FF, et al. Longevity and associated risk factors in adhesive restorations of young permanent teeth after complete and selective caries removal: a retrospective study. Clin Oral Investig. 2016; [Epub ahead of print]. doi: 10.1007/s00784-016-1832-1 
30. World Health Organization. The Ottawa

Charter for Health Promotion. Geneva,

Switzerland: WHO; 1986. 